\title{
Corrigendum to "Williams-Beuren Syndrome: A Clinical Study of 55 Brazilian Patients and the Diagnostic Use of MLPA"
}

\author{
Rachel Sayuri Honjo, ${ }^{1}$ Roberta Lelis Dutra, ${ }^{2}$ Erika Arai Furusawa, \\ Evelin Aline Zanardo, ${ }^{2}$ Larissa Sampaio de Athayde Costa, ${ }^{1}$ Leslie Domenici Kulikowski, ${ }^{2}$ \\ Debora Romeo Bertola, ${ }^{1}$ and Chong Ae Kim ${ }^{1}$ \\ ${ }^{1}$ Clinical Genetics Unit, Instituto da Criança, Hospital das Clínicas, Faculdade de Medicina, Universidade de São Paulo, \\ 05403-000 São Paulo, SP, Brazil \\ ${ }^{2}$ Department of Pathology, Laboratório de Citogenômica, LIM 03, Hospital das Clínicas, Faculdade de Medicina, \\ Universidade de São Paulo, 05403-000 São Paulo, SP, Brazil \\ ${ }^{3}$ Department of Nephrology, Instituto da Criança, Hospital das Clínicas, Faculdade de Medicina, Universidade de São Paulo, \\ 05403-000 São Paulo, SP, Brazil
}

Correspondence should be addressed to Rachel Sayuri Honjo; rachel.honjo@gmail.com

Received 14 September 2015; Accepted 20 September 2015

Copyright (C) 2015 Rachel Sayuri Honjo et al. This is an open access article distributed under the Creative Commons Attribution License, which permits unrestricted use, distribution, and reproduction in any medium, provided the original work is properly cited.

In the paper titled "Williams-Beuren Syndrome: A Clinical Study of 55 Brazilian Patients and the Diagnostic Use of MLPA" [1], the following section is added.

\section{Acknowledgment}

This work was supported by grants from Fundação de Amparo à Pesquisa do Estado de São Paulo (FAPESP) (2010/52710-3; 2015/50022-6).

\section{References}

[1] R. S. Honjo, R. L. Dutra, E. A. Furusawa et al., "WilliamsBeuren syndrome: a clinical study of 55 Brazilian patients and the diagnostic use of MLPA," BioMed Research International, vol. 2015, Article ID 903175, 6 pages, 2015. 

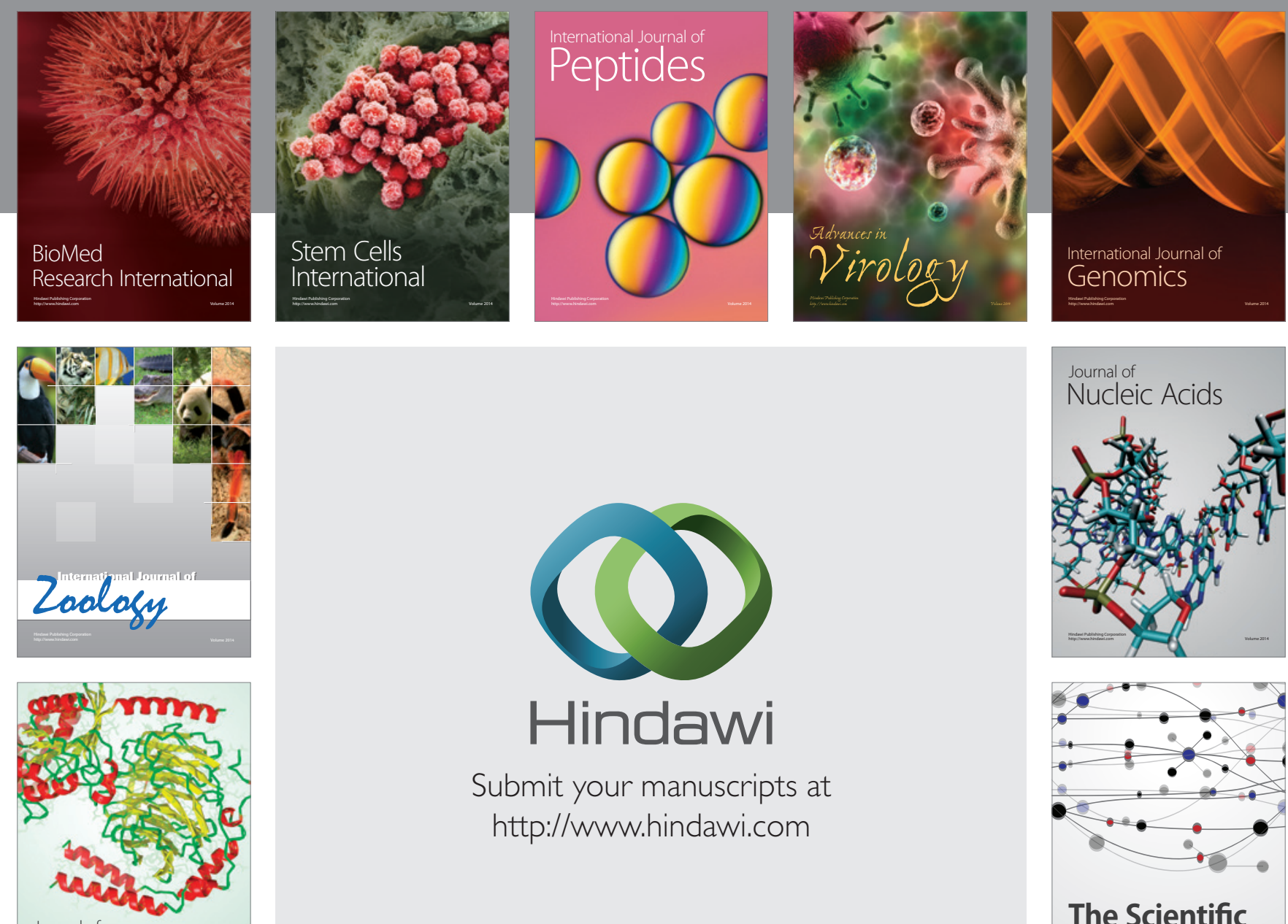

Submit your manuscripts at

http://www.hindawi.com

Journal of
Signal Transduction
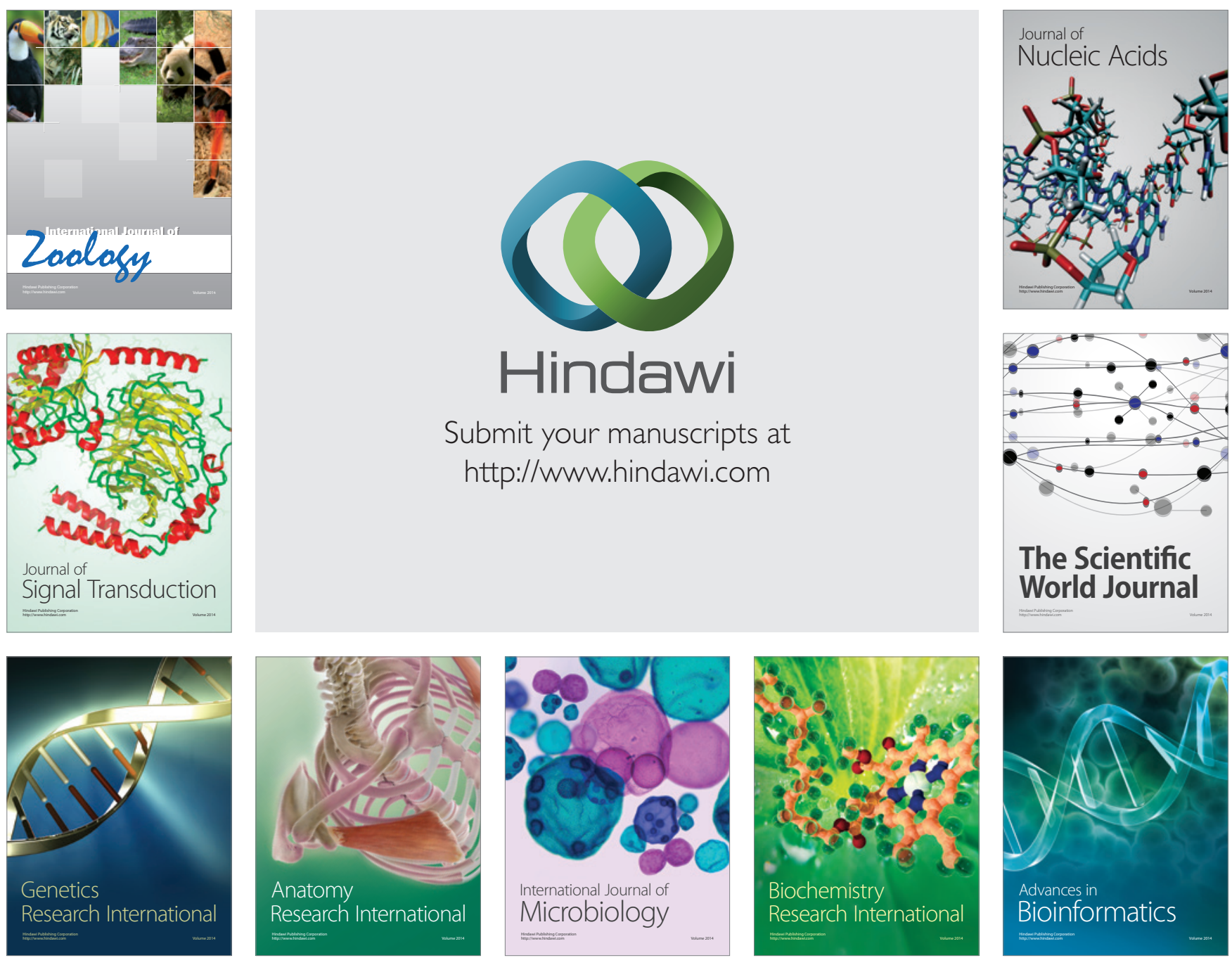

The Scientific World Journal
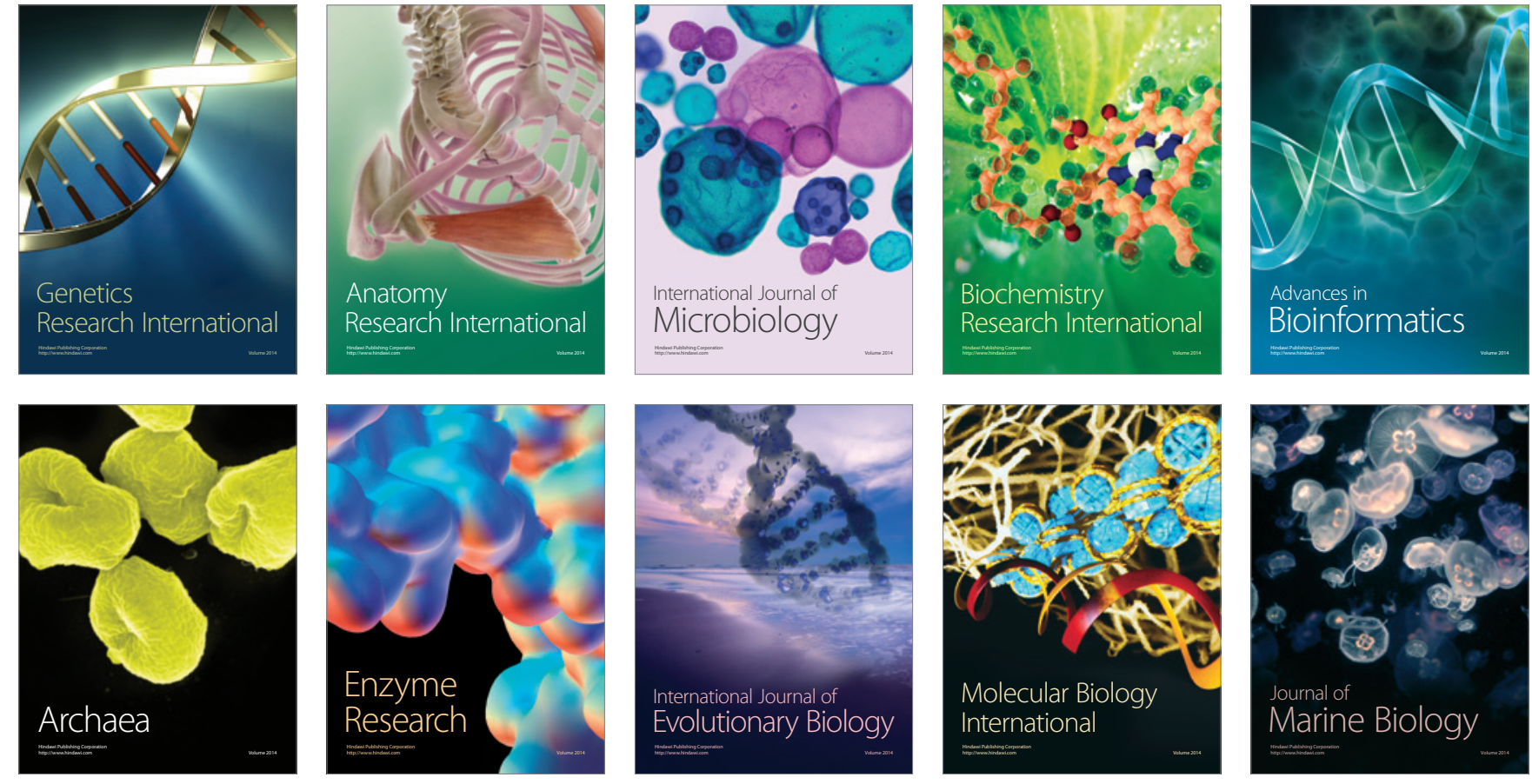